\title{
A Simple Analytic Approximation of Luminosity Distance in FLRW Cosmology using Daftardar-Jafari Method
}

\author{
V. K. Shchigolev \\ Department of Theoretical Physics, Ulyanovsk State University, Ulyanovsk, 432000, Russia
}

Received April 10, 2020; Revised May 15, 2020; Accepted May 27, 2020

Copyright $\odot 2020$ by authors, all rights reserved. Authors agree that this article remains permanently open access under the terms of the Creative Commons Attribution License 4.0 International License

\begin{abstract}
In this paper, the iterative method suggested by Daftardar-Gejji and Jafari hereafter called Daftardar-Jafari method (DJM) is applied for the approximate analytical representation of the luminosity distance in a homogenous Friedmann-Lemaître-Robertson-Walker (FLRW) cosmology. We obtain the analytical expressions of the luminosity distance using the approximate solutions of the differential equation to which the luminosity distance satisfies, subject to the corresponding initial conditions. With the help of this approximate solution, a simple analytic formula for the luminosity distance as a function of redshift is obtained and compared with a numerical solution for the general integral formula by the Maple software. Subsequent comparison of the obtained approximate analytical formula with the corresponding numerical solution for the $\Lambda \mathrm{CDM}$ and quintessential models is provided and showed a high accuracy of the DJM approximations, at least for the certain values of parameters of the models. This comparison demonstrates the efficiency and simplicity of this approach to the problem of calculating the luminosity distance in theoretical cosmology.
\end{abstract}

Keywords FLRW Cosmology, Luminosity Distance, Redshift, Daftardar-Jafari Iterative Method

\section{Introduction}

The database, obtained in the observational astronomy on Supernovae of type Ia as one of the best cosmological distance indicators [1]-[3], encourages theoretical cosmologists to a strong restriction of the essential parameters in their cosmological models. The reason is that these cosmological observations clearly prove a spatial flatness and the present acceleration of our Universe. As a result, the SNIa Union2 database [4] becomes one of the most reliable observational resources for testing various cosmological models. To realize such testing, a researcher has to use the maximum-likelihood approach minimizing $\chi^{2}$ which measures the deviations of the theoretical predictions from the observations. Since SN Ia behave as excellent standard candles, they can be used to directly measure the expansion rate of the Universe. The SN Ia database gives us the distance modulus $\mu$ to each SN Ia supernova. In a flat universe, the theoretical distance modulus is given by

$$
\mu(z)=5 \log _{10}\left(d_{L} / M p c\right)+25
$$

where $d_{L}$ is the luminosity distance. It depends on the cosmological redshift $z$ and the model parameters. Thus, the analytical calculation of the luminosity distance $d_{L}$ versus cosmological redshift $z$ seems to be a very important issue in theoretical cosmology.

Unfortunately, the the luminosity distance formula is usually expressed by an integral over the redshift. As a rule, this integration cannot be prepared explicitly. Moreovere, the latest data on supernovae Ia can involve the redshift $z>2$, that can give rise to a theoretical question about the convergence of approximate solutions for large redshift. Therefore, one has to find some reliable analytic computing procedure for the luminosity distance as a function of redshift.

It could be mentioned that a simple algebraic approximation for the luminosity distance is considered in [5]. Besides, the so-called Padé approximant is applied for the analytical approximation of the luminosity distance in $[6,7,8]$, and in [9] it is shown that the integral in general formula for the luminosity distance can be partly calculated analytically with the help of elliptic integral of the first kind. An interesting numerical strategies of computing the luminosity distance is developed in [10].

Recently, an interesting examples of approximate calculation of the luminosity distance via the Homotopy Perturbation Method (HPM) [11] and the Variational Iteration Method (VIM) [12] have been proposed in Refs. [13] and [14].

The Daftardar-Jafari method (DJM) developed by DaftardarGejji and Jafari [15] in 2006 has been used by many researchers for solving a variety of linear and nonlinear ordinary and partial differential equations and integral equations (see, for example [16]-[20] and references therein). The method converges to the 
exact solution if it exists through successive approximations. For concrete problems, a few number of approximations can be used for numerical purposes with high degree of accuracy. The DJM does not require any restrictive assumptions for nonlinear terms. It is very effective and reliable, and the solution is obtained in the form of a rapidly convergent series with easily computed components [21]. Moreover, the comparison among DJM, Adomian decomposition method, HPM, and VIM shows the efficiency and high accuracy of DJM for the solution of the high order nonlinear boundary value problems $[22,23]$. It would be useful to note a new approach to perturbation method, such as the optimal perturbation iteration method (OPIM) for solving the nonlinear differential equations (see, for example, [24]-[26] and references therein ). The results of these papers reveal that the new approximate solutions obtained via OPIM applied to several problems are even more accurate and impressive than many other techniques in literature.

In this paper, we use the idea of approximate analytical calculation of the luminosity distance by virtue of solving the corresponding differential equation with certain initial conditions, proposed in [13]. Solving this equation in a spatially flat FLRW universe by means of DJM, we obtain the approximate analytical expressions for the luminosity distance in terms of redshift. We show that by using the DJM, the expression for $d_{L}(z)$ in arbitrary accuracy can be easily obtained by implementing a simple procedure for the governing equation.

\section{A Brief Description of DJM}

In order to introduce the basic idea of DJM [15] for solving nonlinear differential equations, we briefly recall the general approach and its application to the second order differential equations.

\subsection{The main idea of DJM}

Let us consider the following general functional equation:

$$
u=N(u)+f
$$

where $N$ is a nonlinear operator from a Banach space $B \rightarrow B$ and $f$ is a known function. We are looking for a solution $u$ of Eq. (1) having the series form:

$$
u=\sum_{i=0}^{\infty} u_{i}
$$

The nonlinear operator $N$ can be decomposed as

$$
N\left(\sum_{i=0}^{\infty} u_{i}\right)=N\left(u_{0}\right)+\sum_{i=0}^{\infty}\left[N\left(\sum_{j=0}^{i} u_{j}\right)-N\left(\sum_{j=0}^{i-1} u_{j}\right)\right]
$$

As it follows from Eqs. (2) and (3), Eq. (1) is equivalent to

$$
\sum_{i=0}^{\infty} u_{i}=f+N\left(u_{0}\right)+\sum_{i=0}^{\infty}\left[N\left(\sum_{j=0}^{i} u_{j}\right)-N\left(\sum_{j=0}^{i-1} u_{j}\right)\right]
$$

One could define the following recurrence relation:

$$
\left\{\begin{array}{c}
u_{0}=f \\
u_{1}=N\left(u_{0}\right), \\
u_{m+1}=N\left(u_{0}+\ldots+u_{m}\right)-N\left(u_{0}+\ldots+u_{m-1}\right),
\end{array}\right.
$$

where $m=1,2, \ldots$.

Then

$$
\left(u_{1}+\ldots+u_{m+1}\right)=N\left(u_{0}+\ldots+u_{m}\right), m=1,2, \ldots,
$$

and

$$
u=f+\sum_{i=1}^{\infty} u_{i} .
$$

The $m$-term approximate solution of Eq. (2) is given by $u=$ $u_{0}+u_{1}+\ldots+u_{m-1}$. If $N$ is a contraction, i.e. $\| N(x)-$ $N(y)\|\leq q\| x-y \|, \quad 0<q<1$, then

$$
\begin{array}{r}
\left\|u_{m+1}\right\|=\| N\left(u_{0}+\ldots+u_{m}\right)-N\left(u_{0}+\ldots+u_{m-1} \|\right. \\
\leq q\left\|u_{m}\right\| \leq q^{m}\left\|u_{0}\right\|, \quad m=0,1,2, \ldots
\end{array}
$$

and the series $\sum_{i=1}^{\infty} u_{i}$ in (7) absolutely and uniformly converges to a solution of Eq. (1), which is unique, in view of the Banach fixed point theorem [15]. For more details about the convergence of the DJM, we refer the reader to Ref. [21].

\subsection{Solving a second order differential equation by using DJM}

Here our description mainly follows to Ref.[23]. Consider some non-linear ordinary differential equation of the second order,

$$
u^{\prime \prime}(z)+k_{1} u^{\prime}(z)+k_{2} u(z)+\tilde{N}(u)=\tilde{f}(z),
$$

where a prime stands for derivative with respect to $z, k_{1}, k_{2}$ are arbitrary constants, $\tilde{f}(z)$ is a given continuous function, and $\tilde{N}(u)$ is a non-linear term. Besides, this equation must satisfy the initial condition:

$$
u(0)=A, u^{\prime}(0)=B .
$$

Equation (8) can be written in an operator form as:

$$
L_{z z} u(z)+k_{1} L_{z} u(z)+k_{2} u(z)+\tilde{N}(u)=\tilde{f}(z),
$$

where $L_{z}=\frac{d}{d z}$ and $L_{z z}=\frac{d^{2}}{d z^{2}}$. We assume that the inverse operators $L_{z}^{-1}$ and $L_{z z}^{-1}$ exist and can be taken as follows

$$
L_{z}^{-1}(.)=\int_{0}^{z}(.) d s
$$

and

$$
L_{z z}^{-1}(.)=\int_{0}^{z} d s \int_{0}^{s}(.) d t=\int_{0}^{z}(z-s)(.) d s
$$


where we have used the Cauchy formula for repeated integration:

$\int_{0}^{z} \int_{0}^{s_{1}} \ldots \int_{0}^{s_{n-1}} \phi\left(s_{n}\right) d s_{n} \ldots d s_{2} d s_{1}=\frac{1}{(n-1) !} \int_{0}^{z}(z-s)^{n-1} \phi(s) d s$,

Then, applying the inverse operator $L_{z z}^{-1}$ to both sides of the equation (10) and taking into account the initial condition (9), we have

$$
\begin{aligned}
u(z)=A+k_{1} A z & +B z+g(z)-L_{z}^{-1} k_{1} u(z) \\
& -L_{z z}^{-1}\left[k_{2} u(z)+\tilde{N}(u(z))\right],
\end{aligned}
$$

where

$$
g(z)=\int_{0}^{z} d s \int_{0}^{s} \tilde{f}(t) d t=\int_{0}^{z}(z-s) \tilde{f}(s) d s .
$$

Therefore, by using equations (11)-(15), we can represent equation (14) in the form of equation (1) by setting

$$
f(z)=A+k_{1} A z+B z+\int_{0}^{z}(z-s) \tilde{f}(s) d s,
$$

and

$$
\begin{aligned}
& N(u)=-L_{z}^{-1} k_{1} u(z)-L_{z z}^{-1}\left[k_{2} u(z)+\tilde{N}(u(z))\right] \\
& =-\int_{0}^{z}\left(k_{1} u(s)+(z-s)\left[k_{2} u(s)+\tilde{N}(u(s))\right]\right) d s .
\end{aligned}
$$

By using expressions (16) and (17) in equation (1), we can follow the procedure (5) in order to obtain solution (7) of ODE (8), provided (9).

Thereafter, we are going to apply DJM in the problem of approximation for the luminosity distance in FLRW cosmology.

\section{Formulation of the problem}

A homogeneous isotropic universe can be described by the following FLRW metrics [27],

$$
d s^{2}=-d t^{2}+a^{2}(t)\left[\frac{d r^{2}}{1-k r^{2}}+r^{2}\left(d \theta^{2}+\sin \theta d \phi^{2}\right)\right]
$$

where $a(t)$ is a scale factor, and $k=1,0,-1$ for a closed, spatially-flat, open universe respectively, and the speed of light in vacuum $c=1$. The Einstein equation of General Relativity in the cosmological model (18) with a cosmological constant $\Lambda$, leads to the first Friedmann equation as follows

$$
\left(\frac{H}{H_{0}}\right)^{2}=\sum_{m} \Omega_{m} a^{-3\left(1+w_{m}\right)}+\Omega_{k} a^{-2}+\Omega_{\Lambda},
$$

where $H=\frac{\dot{a}}{a}$ is the Hubble parameter, $H_{0}$ denotes its present value, and the dimensionless densities are

$$
\Omega_{m}=\frac{8 \pi G}{3 H_{0}^{2}} \rho_{m}, \quad \Omega_{k}=-\frac{k}{H_{0}^{2} a_{0}^{2}}, \quad \Omega_{\Lambda}=\frac{\Lambda}{3 H_{0}^{2}},
$$

where $G$ is Newton's gravitational constant, $\Omega_{\Lambda}$ is the contribution of vacuum, $\Omega_{k}$ is the contribution associated with curvature, and $\Omega_{m}$ is the contribution of all other kinds of matter and fields with the equation of state parameters $(\mathrm{EoS}) w_{m}$ and the energy density $\rho_{m}$. From equation (20), it follows that these parameters satisfy

$$
\sum_{m} \Omega_{m}+\Omega_{k}+\Omega_{\Lambda}=1
$$

One of the most fundamental distance scale in relativistic cosmology is the luminosity distance. It is defined by $d_{L}=$ $\sqrt{\frac{L}{4 \pi f}}$, where $f$ is the observed flux of an astronomical object and $L$ is its luminosity. According to the recent astronomical observations $[1,2]$, our universe is spatially flat, and the present density parameter $\Omega_{\Lambda} \approx 0.72$.

As known [27], the luminosity distance $d_{L}$ of a spatially flat universe is as follows

$$
d_{L}=\frac{c(1+z)}{H_{0}} \int_{o}^{z} \frac{d z^{\prime}}{\sqrt{W\left(z^{\prime}\right)}}
$$

where $W(z)=\left(\frac{H(z)}{H_{0}}\right)^{2}$ depends on the Hubble parameter $H(z)$ as a function of redshift $z=\frac{a_{0}}{a}-1$, which obeys equation (19). Due to equations (19) and (21), we get

$$
W(z)=\sum_{m} \Omega_{m}(1+z)^{3\left(1+w_{m}\right)}+\Omega_{\Lambda},
$$

that is

$$
W(0) \equiv W(z){ }_{\mid z=0}=1
$$

For example, one has

$$
W(z)=\Omega_{m}(1+z)^{3}+\Omega_{r}(1+z)^{4}+\Omega_{\Lambda}
$$

in the $\Lambda$ CDM model of the universe [27], where $\Omega_{m}, \Omega_{r}$ and $\Omega_{\Lambda}$ are the energy densities corresponding to the dust-like matter, radiation and cosmological constant, respectively. According to (24), we have $\Omega_{m}+\Omega_{r}+\Omega_{\Lambda}=1$. Even this simple example demonstrates that the explicit calculation of the integral in equation (22) is rather problematic. In general, this calculation often involves some numerical calculations, elliptic functions or algebraic approximations.

In our previous works $[13,14]$, we proposed to apply two approximate methods for calculating the luminosity distance as a solution of the Cauchy problem for the differential equation followed from (22). By introducing a new unknown function

$$
u(z)=\frac{H_{0} d_{L}}{c(1+z)}
$$

we can rewrite equation (22) in the following form

$$
u(z)=\int_{o}^{z} \frac{d z^{\prime}}{\sqrt{W\left(z^{\prime}\right)}}
$$


Thus, the derivatives of $u(z)$ are as follows

$$
\frac{d u}{d z}=\frac{1}{\sqrt{W}}, \quad \frac{d^{2} u}{d z^{2}}=-\frac{1}{2} W^{-\frac{3}{2}} \frac{d W}{d z} .
$$

Combining these two equations, we obtain the following main equation

$$
\frac{d^{2} u}{d z^{2}}=-\frac{1}{2} W^{\prime}\left(\frac{d u}{d z}\right)^{3}
$$

where and below the prime stands for the derivative with respect to $z$. In addition, equations (24), (27) and (28) yield the following initial conditions for $u(z)$

$$
\left.u(z)\right|_{z=0}=0,\left.\frac{d u}{d z}\right|_{z=0}=1 .
$$

\section{Luminosity Distance in DJM Ap- proximation}

The Cauchy problem (29), (30) for the nonlinear differential equation of the second order can be solved exactly in quadratures, but the result leads to the integral (27). Therefore, we can try to solve this problem analytically with a certain approximation using some method, e.g. DJM .

Comparing the equations (8), (9) with the corresponding equations (29), (30) gives us the following equalities:

$$
k_{1}=k_{2}=0, \quad A=0, \quad B=1,
$$

and

$$
\tilde{f}=0, \quad \tilde{N}=\frac{1}{2} W^{\prime}(z)\left(u^{\prime}(z)\right)^{3} .
$$

Using the equations (31), (32) in (16) and (17), we get

$$
f(z)=z, N(u)=-\frac{1}{2} \int_{0}^{z}(z-s) W^{\prime}(s)\left(u^{\prime}(s)\right)^{3} d s .
$$

Therefore, the functional equation (1) becomes as follows

$$
u(z)=z-\frac{1}{2} \int_{0}^{z}(z-s) W^{\prime}(s)\left(u^{\prime}(s)\right)^{3} d s .
$$

Starting with the initial approximation $u_{0}(z)=z$ according to (5) and (33), one can obtain the next term,

$$
u_{1}(z)=N\left(u_{0}\right)=-\frac{1}{2} \int_{0}^{z}(z-s) W^{\prime}(s) d s,
$$

in the m-term approximate solution (7) of the following form

$$
u(z)=z+\sum_{i=1}^{m-1} u_{i}(z)
$$

where $f(z)=z$ due to (33). Let us find $u_{2}(z)$ as

$$
u_{2}(z)=N\left(u_{0}+u_{1}\right)-N\left(u_{0}\right)
$$

due to (5). Using equations (33) and (35) in (37), we get

$$
u_{2}(z)=\frac{1}{2} \int_{0}^{z}(z-s) W^{\prime}(s)\left[1-\frac{(3-W(s))^{3}}{8}\right] d s,
$$

where the equations (24), (35) and

$$
\left(u_{0}(s)+u_{1}(s)\right)^{\prime}=\frac{3}{2}-\frac{W(s)}{2}
$$

are used. Then taking into account equations (35), (38) and integrating by parts, we can express the second-order approximation for the luminosity distance (26) as follows

$$
d_{L}(z)=\frac{c(1+z)}{H_{0}}\left[\frac{3}{4} z+\frac{1}{64} \int_{0}^{z}(3-W(s))^{4} d s\right] .
$$

One can see that the approximate formula (39) can be readily applied not only in the models with a polynomial dependence of the Hubble parameter squared over $(1+z)$, but also to many other FRW models when the analytic calculation of the integral in (22) becomes fairly problematic. The DJM approximation obtained here gives an analytic expression for $d_{L}(z)$ in a rather good accuracy.

\section{Two Examples of DJM Approxima- tion}

In the first instance, we consider a simple example of the luminosity distance in $\Lambda$ CDM model of the universe (22) where $W(z)$ is given by $(25)$, that is

$$
d_{L}(z)=\frac{c(1+z)}{H_{0}} \int_{0}^{z} \frac{d z^{\prime}}{\sqrt{\Omega_{r}\left(1+z^{\prime}\right)^{4}+\Omega_{m}\left(1+z^{\prime}\right)^{3}+\Omega_{\Lambda}}} .
$$

According to the modern observations, the present radiation density is relatively very small, $\Omega_{r} \sim 10^{-4}$, and this term in (40) can be neglected. Therefore, we get

$$
W(z)=\Omega_{m}(1+z)^{3}+\Omega_{\Lambda},
$$

where $\Omega_{\Lambda}=1-\Omega_{m}$. Substituting (41) in (39), one can obtain the following approximation for the luminosity distance in $\Lambda \mathrm{CDM}$ model:

$$
\begin{aligned}
& d_{L}(z)= \frac{c}{H_{0}}(z+1)\left[\frac{3}{4} z+\frac{1}{832} \Omega_{m}^{4}\left((z+1)^{13}-1\right)\right. \\
&-\frac{1}{160}\left(2+\Omega_{m}\right) \Omega_{m}^{3}\left((z+1)^{10}-1\right) \\
&+\frac{3}{224}\left(2+\Omega_{m}\right)^{2} \Omega_{m}^{2}\left((z+1)^{7}-1\right) \\
&\left.-\frac{1}{64}\left(2+\Omega_{m}\right)^{3} \Omega_{m}\left((z+1)^{4}-1\right)+\frac{1}{64}\left(2+\Omega_{m}\right)^{4} z\right] .
\end{aligned}
$$

One can compare this approximation with the well known expansion of luminosity distance $d_{L}$ given by a Taylor series in redshift $z$, that is (see, e.g., [28])

$d_{L}(z)=\frac{c z}{H_{0}}\left[1+\frac{1}{2}\left(1-q_{0}\right) z-\frac{1}{6}\left(1-q_{0}-3 q_{0}^{2}+j_{0}\right) z^{2}+O\left(z^{3}\right)\right]$, 


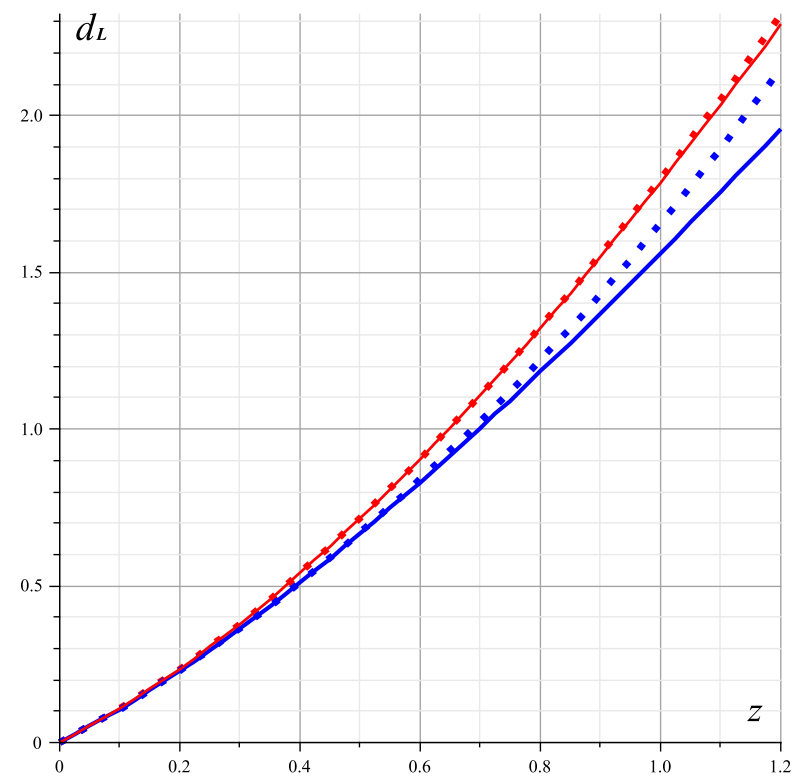

Figure 1. Comparison of the numerical solution to the integral in equation (40) (solid lines) and the approximate solution (42) (point lines) for the $\Lambda$ CDM model with $\Omega_{m}=0.28, \Omega_{\Lambda}=0.72, \Omega_{r}=0, \Omega_{r}=0$ (in blue) or $\Omega_{m}=$ $0.10, \Omega_{\Lambda}=0.90, \Omega_{r}=0$ (in red). Here, $c / H_{0}=1$.

where $q_{0}$ and $j_{0}$ stand for the present magnitudes of the deceleration parameter $q=-\frac{a \ddot{a}}{\dot{a}^{2}}$ and the jerk parameter $j=\frac{a^{2} \dddot{a}}{\dot{a}^{3}}$, respectively. By using (43), it is easy to show that approximate solution (42) yields the following expression for $q_{0}$

$$
q_{0}=-1+\frac{3}{2} \Omega_{m}
$$

If we insert $\Omega_{\Lambda}=0.72$ and $\Omega_{m}=0.28$ in (29) for the illustrative purpose, then we have $q_{0} \approx-0.58$, which is within the observational limits.

Table 1. Percentage of relative errors of the approximate $d_{L}$ given by Eq. (42) with $\Omega_{\Lambda}=0.72$ (Case 1) and $\Omega_{\Lambda}=0.90$ (Case 2).

\begin{tabular}{|c||l|l|}
\hline$z$ & \% of RE in Case 1 & \% of RE in Case 2 \\
\hline \hline 0.1 & 0.003262 & 0.000156 \\
\hline 0.2 & 0.03011 & 0.00153 \\
\hline 0.3 & 0.11552 & 0.006222 \\
\hline 0.4 & 0.304289 & 0.017632 \\
\hline 0.5 & 0.649655 & 0.040712 \\
\hline 0.6 & 1.204233 & 0.092224 \\
\hline 0.7 & 2.011819 & 0.150852 \\
\hline 0.8 & 3.097237 & 0.257152 \\
\hline 0.9 & 4.457996 & 0.413277 \\
\hline 1.0 & 6.062429 & 0.632454 \\
\hline 1.1 & 7.861499 & 0.928192 \\
\hline 1.2 & 9.825002 & 1.313241 \\
\hline
\end{tabular}

With the help of Maple package, the graphs of $d_{L}(z)$ in units of $c / H_{0}$ for the numerical solutions to the integral in equation (40), and the approximate solution (42) are shown in Fig. 1, where we have used $\Omega_{m}=0.28, \Omega_{\Lambda}=0.72$ or $\Omega_{m}=0.10, \Omega_{\Lambda}=0.90$, along with $\Omega_{r}=0$. Besides, we show the percentage of relative errors of the approximate solutions compared to the numerical one in Table 1, from which one can conclude that the accuracy of our approximation is quite acceptable. Indeed, for the range $0 \leq z \leq 1.1$ in Case 2, the relative error is less than $1.0 \%$.

For the next example, we consider a spatially flat universe with the quintessential matter. Let the equation of state parameter be equals to $-1<w_{q}<-1 / 3$. Then equation (23) becomes as follows

$$
W(z)=\Omega_{q}(1+z)^{3\left(1+w_{q}\right)}+\Omega_{\Lambda} .
$$

where $\Omega_{q}$ is the quintessence energy densities. Being substituted into (22), it yields

$$
d_{L}(z)=\frac{c(1+z)}{H_{0}} \int_{0}^{z} \frac{d z^{\prime}}{\sqrt{\Omega_{q}\left(1+z^{\prime}\right)^{3\left(1+w_{q}\right)}+\Omega_{\Lambda}}},
$$

provided that $\Omega_{q}+\Omega_{\Lambda}=1$.

Using $W(z)$ given by (45) in equation (39), we can obtain the following approximation for the luminosity distance

$$
\begin{aligned}
d_{L}(z)= & \frac{c(1+z)}{64 H_{0}}\left\{\left[48+\left(2+\Omega_{q}\right)^{4}\right] z\right. \\
& -\frac{4 \Omega_{q}\left(2+\Omega_{q}\right)^{3}}{\left(4+3 w_{q}\right)}\left[(1+z)^{4+3 w_{q}}-1\right] \\
& +\frac{6 \Omega_{q}^{2}\left(2+\Omega_{q}\right)^{2}}{\left(7+6 w_{q}\right)}\left[(1+z)^{7+6 w_{q}}-1\right] \\
& -\frac{4 \Omega_{q}^{3}\left(2+\Omega_{q}\right)}{\left(10+9 w_{q}\right)}\left[(1+z)^{10+9 w_{q}}-1\right] \\
& \left.+\frac{\Omega_{q}^{4}}{\left(13+12 w_{q}\right)}\left[(1+z)^{13+12 w_{q}}-1\right]\right\} .
\end{aligned}
$$

Formally, equations (45)-(47) are valid for any states of barotropic matter. For example, one can easily verify that in the case of dust, i.e. $w_{q}=0$, formula (47) just coincides with formula (42). At the same time, for the vacuum-like state $w_{q}=$ -1 , the result followed from $(47), d_{L}(z)=c H_{0}^{-1}(1+z) z$, is rather obvious, because it can be simply found directly from equation (46).

The graphs of the approximate solution (47) for (i) $\Omega_{q}=$ $0.3, w_{w}=-2 / 3$, and (ii) $\Omega_{q}=0.7, w_{q}=-2.8 / 3$ compared to the numerical solution to the integral in equation (46) in the same cases are shown in Fig. 2. As can be concluded from these graphs, the approximate expression for the luminosity distance (47) gives good results for very different densities of matter in the Universe. The graphs of correspondent percentage of the relative errors given by the approximate solution (47) in cases (i) and (ii) are depicted in Fig. 3. From these graphs, we could conclude that the simple approximation (47) may be highly accurate for the model with a certain values of parameters (similar to case (ii)). One can see that a very high degree 


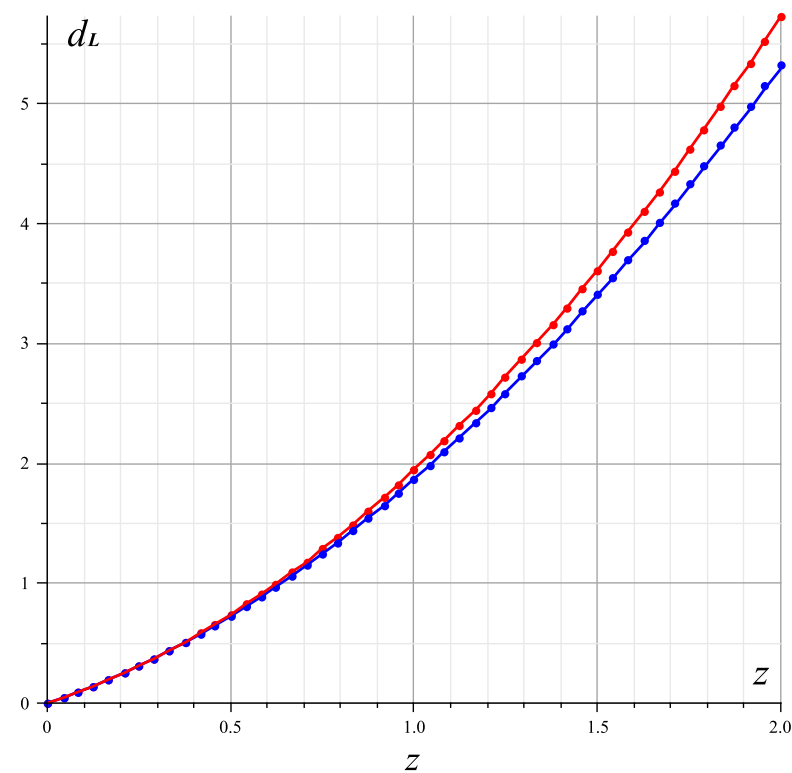

Figure 2. Comparison of the numerical solution to the integral in equation (46) (solid lines) and the approximate solution (47) (point lines) for $\Omega_{q}=$ $0.3, w_{w}=-2 / 3$ (in blue) or $\Omega_{q}=0.7, w_{q}=-2.8 / 3$ (in red). Here, $c / H_{0}=1$.

of accuracy (less than $0.05 \%$ ) is achieved with a percentage increase in the contribution of quintessence to the total mass of matter.

\section{Conclusions}

I this paper, a simple analytical approximation for the luminosity distance in relativistic cosmology via the Daftardar-Jafari method has been obtained. For this purpose, the problem of calculating the integral in the expression for the luminosity distance (22) has been transformed into the Cauchy problem for the corresponding nonlinear differential equation (29). Thereafter, this equation has been solved by using the approximate analytic method, viz. DJM, with a certain accuracy. Subsequent comparison of the obtained approximate analytical formula (39) with the corresponding numerical solution for the $\Lambda \mathrm{CDM}$ and quintessential models (see Fig. 1 and Fig.2) clearly showed a high accuracy of the DJM approximations, at least for the certain values of their parameters. The latter can be seen from Tab. 1 and Fig. 3. The obvious advantage of the formula (39) is that this approximation does not initially involves a Taylor series expansion in the redshift or in some physical parameter. Nevertheless, even a few number of iteration steps leads to a high accuracy for the analytical approximation of the luminosity distance. It should be emphasized that this approximation for the luminosity distance can be applied to the variety of cosmological models with different expressions for $W(z)$, even to the models with a non-polynomial over $(1+z)$ function $W(z)$. Therefore, one can conclude that the DJM is a powerful and efficient technique to solve the problem of the luminosity distance computation in theoretical cosmology.

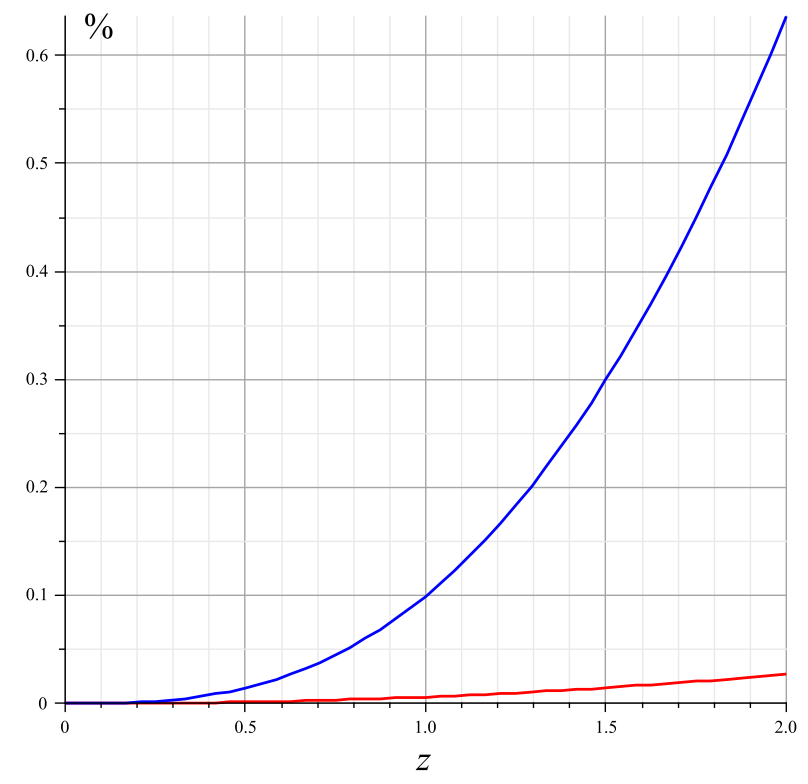

Figure 3. Percentage of the relative errors given by the approximate solution (47) with $\Omega_{q}=0.3, w_{w}=-2 / 3$ (in blue) or $\Omega_{q}=0.7, w_{q}=-2.8 / 3$ (in red).

\section{REFERENCES}

[1] A. G. Riess, et al. Observational Evidence from Supernovae for an Accelerating Universe and a Cosmological Constant, Astronomical Journal, Vol. 116, 1009, 1998. http://dx.doi.org/10.1086/300499

[2] S. Perlmutter, et al. Measurements of Omega and Lambda from 42 High-Redshift Supernovae, Astrophysical Journal, Vol. 517, 565, 1999. http://dx.doi.org/10.1086/307221

[3] N. Suzuki, D. Rubin, C. Lidman, et al. The Hubble Space Telescope Cluster Supernova Survey. V. Improving The DarkEnergy Constraints Above $z>1$ and Building an EarlyType-Hosted Supernova Sample, Astrophysical Journal, 746, 85, 2012. http://dx.doi.org/10.1088/0004-637X/746/1/85

[4] R. Amanullah, et al. Spectra and Light Curves of Six Type Ia Supernovae at $0.511<z<1.12$ and the Union2 Compilation, Astrophysical Journal, Vol. 716, 712-738, 2010. http://dx.doi.org/10.1088/0004-637X/716/1/712

[5] Ue-Li Pen. Analytical Fit to the Luminosity Distance for Flat Cosmologies with a Cosmological Constant, Astrophys. J. Suppl. S., Vol. 120, 4950, 1999. http://dx.doi.org/10.1086/313167

[6] Masaru Adachi, Masumi Kasai. An Analytical Approximation of the Luminosity Distance in Flat Cosmologies with a Cosmological Constant, Progress of Theoretical Physics, Vol. 127, Issue 1, 145-152, 2012. https://doi.org/10.1143/PTP.127.145

[7] Hao Wei, Xiao-Peng Yan, Ya-Nan Zhou. Cosmological Applications of Pade Approximant, JCAP, Vol. 1401, 045, 2014. http://dx.doi.org/10.1088/1475-7516/2014/01/045 
[8] L. Zaninetti. Pade Approximant and Minimax Rational Approximation in Standard Cosmology, Galaxies, 4(1), 4, 2016. https://doi.org/10.3390/galaxies4010004

[9] A. Meszaros, J. Ripa. On the relation between the non-flat cosmological models and the elliptic integral of first kind, Astron. Astrophys., Vol. 573, A54, 2015. https://doi.org/10.1051/0004$6361 / 201425201$

[10] De-Zi Liu, Cong Ma, Tong-Jie Zhang, and Zhiliang Yang. Numerical Strategies of Computing the Luminosity Distance, Mon. Not. R. Astron. Soc., Vol. 412, 2685, 2011. http://dx.doi.org/10.1111/j.1365-2966.2010.18101.x

[11] J.-H. He. Homotopy perturbation technique, Comput. Meth. Appl. Mech. Eng., Vol. 178, 257?62, 1999. http://dx.doi.org/10.1016/S0045-7825(99)00018-3

[12] J.-H. He. Variational iteration method - a kind of non-linear analytical technique: some examples, International Journal of Non-Linear Mechanics, Vol. 34, No. 4, 699-708, 1999. http://dx.doi.org/10.1016/S0020-7462(98)00048-1

[13] V. K. Shchigolev. Calculating Luminosity Distance versus Redshift in FLRW Cosmology via Homotopy Perturbation Method, Gravit. Cosmol., 23: 142, 2017. http://dx.doi.org/10.1134/S0202289317020098.

[14] V. K. Shchigolev. Analytic Approximation of Luminosity Distance in Cosmology via Variational Iteration Method, Universal Journal of Computational Mathematics, 5(3): 68-74, 2017. http://dx.doi.org/10.13189/ujcmj.2017.050303

[15] V. Daftardar-Gejji, H. Jafari. An iterative method for solving non-linear functional equations, Journal of Mathematical Analysis and Applications, 316: 753?63, 2006. http://dx.doi.org/10.1016/j.jmaa.2005.05.009.

[16] Sinan H. Abd Almjeed1, Ghassan H. Radhi. Daftardar-Jafari Method for Solving the Damped Generalized Regularized Long-Wave Equation, International Journal of Science and Research, 6(2): 1863-1867, 2017. Paper ID: ART20179697 DOI: 10.21275/ART20179697

[17] S. Bhalekar and V. Daftardar-Gejji. New Iterative Method: Application to Partial Differential Equations, Applied Mathematics and Computation, 203: 778- 783, 2008. https://doi.org/10.1016/j.amc.2008.05.071

[18] V. Daftardar-Gejji and S. Bhalekar. Solving Fractional Boundary Value Problems with Dirichlet Boundary Conditions Using a New Iterative Method, Computers \&
Mathematics with Applications, 59: 1801-1809, 2010. https://doi.org/10.1016/j.camwa.2009.08.018

[19] V. Daftardar-Gejji and S. Bhalekar. An Iterative Method for Solving Fractional Differential Equations, Proceedings in Applied Mathematics and Mechanics, 7: 2050017-2050018, 2008. https://doi.org/10.1002/pamm.200701001

[20] S. Bhalekar and V. Daftardar-Gejji. Solving Evolution Equations Using a New Iterative Method, Numerical Methods for Partial Differential Equations, 26: 906-916, 2010. https://doi.org/10.1002/num.20463

[21] S. Bhalekar, V. Daftardar-Gejji. Convergence of the New Iterative Method, International Journal of Differential Equations, 2011, Arti-cle ID 989065, 10 pages. http://dx.doi.org/10.1155/2011/989065

[22] I. Ullah, H. Khan, and M. T. Rahim. Numerical Solutions of Fifth and Sixth Order Nonlinear Boundary Value Problems by Daftardar Jafari Method, Journal of Computational Engineering Volume 2014, Article ID 286039, 8 pages. http://dx.doi.org/10.1155/2014/286039

[23] M. A. Al-Jawary, S. G. Abd-Al-Razaq. Analytic and numerical solution for Duffing equations, International Journal of Basic and Applied Sciences, 5 (2): 115-119, 2016. http://dx.doi.org/10.14419/ijbas.v5i2.5838

[24] N. Bildik and S. Deniz. A new efficient method for solving delay differential equations and a comparison with other methods, The European Physical Journal Plus, 132(1), 51, 2017. http://dx.doi.org/10.1140/epjp/i2017-11344-9

[25] N. Bildik and S. Deniz. New approximate solutions to the nonlinear Klein-Gordon equations using perturbation iteration techniques, Discrete \& Continuous Dynamical Systems-S, 13(3), 503, 2020. http://dx.doi.org/10.3934/dcdss.2020028

[26] Necdet Bildik and Sinan Deniz. New approximate solutions to electrostatic differential equations obtained by using numerical and analytical methods, Georgian Mathematical Journal, 27.1, 23-30, 2020.

[27] S. Weinberg, Gravitation and Cosmology: Principles and Applications of The General Theory of Relativity (John Wiley. Press, New York, 1972).

[28] Takeshi Chiba, Takashi Nakamura. The Luminosity Distance, the Equation of State, and the Geometry of the Universe, Prog. Theor. Phys., Vol.100, 1077, 1998. http://dx.doi.org/10.1143/PTP.100.1077 